\title{
Urtikaria: Neue Empfehlungen zum Management
}

Q uaddeln, Juckreiz und Angioödeme (Abb. 4) sind die Hauptsymptome einer Urtikaria. Die Urtikaria (Abb. 5) gilt als chronisch, wenn die Symptome kontinuierlich oder intermittierend mehr als sechs Wochen andauern. Häufigste Form ist die chronische spontane Urtikaria, das heißt die Quaddeln treten ohne erkennbaren Grund auf. Auslöser einer induzierbaren chronischen Urtikaria ist bei Kindern häufig Kälte, berichtete Dr. Adriane Peveling-Oberhag, Universitäts-Hautklink Mainz. Ein kausaler Zusammenhang zwischen chronischer Urtikaria und dem atopischen Formenkreis besteht nicht.

Die differenzialdiagnostische Abklärung erleichtert ein standardisiertes Diagnoseschema [Maurer $\mathrm{M}$ et al. Allergo J 2013;22(5):324-9]: Kommen bei re-

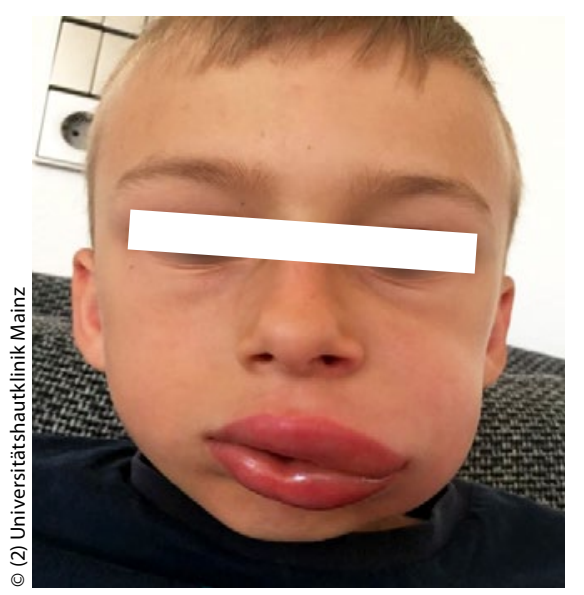

Abb. 4: Angioödem zidivierender Quaddelbildung Fieber, allgemeines Krankheitsgefühl, Knochenoder Gelenkschmerzen hinzu, sollte an eine autoinflammatorische Erkrankung wie CAPS (Cryopyrin-assoziiertes Fiebersyndrom) gedacht werden. Weist das Kind keine weiteren klinischen Krankheitszeichen auf und bestehen die einzelnen Quaddeln länger als 24 Stunden, kann dies auf eine Urtikariavaskulitis hinweisen, die jedoch bei Kindern selten ist. Konnten diese beiden Krankheitsbilder ausgeschlossen werden, ist von einer chronischen Urtikaria auszugehen und zu prüfen, ob eine spontane oder induzierbare Form vorliegt. Bei vermuteten induzierbaren Formen bringt eine Provokationstestung Sicherheit. Treten rezidivierend Angioödeme - ohne Quaddeln - auf, sollte differenzialdiagnostisch ein hereditäres Angioödem abgeklärt werden.

Die Studienlage zur Therapie der chronischen Urtikaria im Kindesalter sei schwach, so Peveling-Oberhag. Die Empfehlungen für das Erwachsenenalter finden auch bei Kindern Anwendung. Therapie der Wahl sind H1-Antihistaminika der 2. Generation, sedierende Antihistaminika sollten vermieden werden. Mit unterschiedlichen Altersbeschränkungen für Kinder zugelassen sind Desloratadin, Cetirizin, Loratadin, Levocetrizin und Rupatadin. Bei mehr als der Hälfte der eigenen Patienten im Kindesalter sei die Symptomkontrolle unter Antihistaminika in Einfachdosis jedoch nicht zufriedenstellend, berichtete die Dermatologin. Persistieren die Sympto-

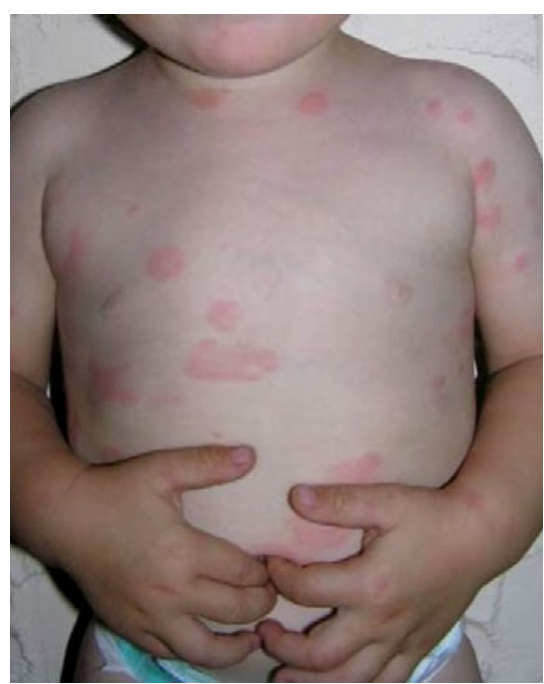

Abb. 5: Urtikaria

me, kann bis zur vierfachen Dosis gesteigert werden. Dies wird von den Kindern in der Regel gut vertragen. Wichtig ist jedoch eine Aufklärung, dass diese Aufdosierung außerhalb der Zulassung erfolgt. Bei fehlendem Ansprechen auf Antihistaminika kommt bei der chronischen spontanen Urtikaria Omalizumab in Frage, das ab einem Alter von 12 Jahren zugelassen ist. Sicherheitsdaten für dieses Medikament liegen im Bereich der Asthmatherapie bereits für Kinder ab 6 Jahren vor. Ein „Wundermittel“ sei Omalizumab zwar nicht, eigenen Erfahrungen zufolge ist es jedoch effektiv und gut verträglich und sollte Kindern mit therapieresistenter chronischer spontaner Urtikaria angeboten werden, sagte Peveling-Oberhag. Angelika Bauer-Delto

Peveling-Oberhag A. Urtikaria bei Kindern Management 2016

\section{Hämangiome: Wann und wie behandeln?}

$\mathrm{E}$ ltern sind oft sehr besorgt, wenn ihr Kind ein Hämangiom aufweist. Doch rund $80 \%$ der Hämangiome im Kindesalter benötigen keine Therapie und bilden sich ohne Komplikationen weitgehend von selbst zurück, betonte Dr. Markus Schneider, Facharzt für Kinder- und Jugendmedizin in Bad Rappenau. Bei Hämangiomen an unproblematischen Lokalisationen sollte abgewartet und der Verlauf überwacht werden. Dabei sollte das Alter des Kindes in Monaten dem Abstand der Kontrolluntersuchungen in Wochen entsprechen.

Eine Therapie sollte bei einer Lokalisation an Körperöffnungen erwogen werden, um Komplikationen vorzubeugen. So kann beispielsweise ein Hämangiom in der Mund-Rachen-Region zu einer Obstruktion der Atemwege oder im Pe- riorbitalbereich zu einer Beeinträchtigung des Sehvermögens führen. Hämangiome an den Ohren sowie im Anogenitalbereich gehen mit einem hohen Risiko für Ulzerationen einher. Bei Hämangiomen an Fingern oder Zehen sollte ebenfalls eine Therapie erwogen werden, da es zu Sensibilitätsstörungen kommen kann. Im Gesichts- oder Brustbereich kann eine kosmetische Indikation gegeben sein, um bleibende Entstellungen zu vermeiden. 